\title{
Patienteninformations-Broschüren
}

\section{in Leichter Sprache}

\author{
Für Menschen mit Leseschwierigkeiten \\ und Problemen beim Verstehen
}

Alexandra Boll

\begin{abstract}
Es gibt zahlreiche Menschen, die beim Lesen und Verstehen von Texten Probleme haben. Ihnen bleibt der Zugang zu wichtigen Informationen zu den Themen Gesundheit und Krankheit verwehrt.

Mit diesem Beitrag soll ein Impuls gegeben werden, sich mit dem Konzept Leichte Sprache zu beschäftigen.
\end{abstract}

In meiner Bachelorarbeit habe ich nach Broschüren in leichter Sprache gesucht, die sich mit Themen zur Gesundheitsprävention und der Behandlung von häufigen Erkrankungen befassen. Für die Pflege und Betreuung von Menschen mit einer geistigen Behinderung ist es wichtig, leicht lesbare und verständliche Texte zur Verfügung zu haben. So können Betroffene entweder sich selbst informieren oder durch Pflegende und Betreuende, (Ärzte und Therapeuten) anhand von Broschüren besser angeleitet und geschult werden. Seit Jahren ist es Standard, durch Patientenedukation Patienten und ihre Familien im Umgang mit Erkrankungen zu informieren, zu schulen, anzuleiten und zu beraten. Ziel ist es, Betroffene im Alltag bei der Bewältigung ihrer eigenen Erkrankung zu unterstützen. Hierzu zählen auch präventive Maßnahmen zur Vorbeugung von Folgeerkrankungen oder Verschlimmerung von Krankheiten.

Leichte Sprache ist laut Universität zu Köln eine „sprachliche Ausdrucksweise die besonders leicht verständlich ist und vor allem Menschen mit geringen sprachlichen Fähigkeiten das Verständnis von Texten erleichtern." (Bredel/Maaß, 2016) Das ,Netzwerk Leichte Sprache" hat folgende Definition veröffentlicht: „Leichte Sprache ist eine sehr leicht verständliche Sprache. Man kann sie sprechen und schreiben." (Netzwerk Leichte Sprache e.V., 2015) Der Ursprung der Leichten Sprache ist in den 60iger Jahren in den USA aufgrund einer Behindertenrechtsbewegung zu finden. Daraus entstanden 1997 die „Guidelines for easyto-readmaterials" - ein erstes Regelwerk für einfach geschriebene Texte. (International Federation of Library Association and Institutions, IFLA Professional Reports, No. 120) In Deutschland wurde im Jahre 2006 das ,Netzwerk
Leichte Sprache' gegründet, welches ebenfalls ein Regelwerk erstellt hat. Außerdem wurde im Jahr 2011 die Barriere freie-Informationstechnik-Verordnung (BITV) in Kraft gesetzt. Sie schreibt die Verfügbarkeit von einfachen Texten für Online-Angebote von Bundesbehörden vor, aufgrund der UN-Behindertenrechtskonvention. (Bundesministerium für Arbeit und Soziales, 2011). Das Regelwerk des ,Netzwerkes Leichte Sprache' scheint in Deutschland und Europa am meisten verbreitet.

In der Literatur zur Leichten Sprache sind als Zielgruppe nicht nur Menschen mit einer geistigen Behinderung, sondern auch Menschen mit einer Demenz, prälingualer Gehörlosigkeit, Aphasie, geringen Deutschkenntnissen sowie Analphabeten genannt. Sie alle können von Informationen in Leichter Sprache profitieren.

In Artikel 25 der Behindertenrechtskonvention wird beschrieben, dass die Menschen, die in den Gesundheitsberufen arbeiten, durch Schulungen für die Arbeit mit behinderten Menschen qualifiziert werden müssen. Außerdem sollen alle geeigneten Maßnahmen getroffen werden, damit Menschen mit Behinderungen das erreichbare Höchstmaß an Gesundheit erlangen können. (Beauftragte der Bundesregierung für die Belange behinderter Menschen, 2014)

Die Ottawa-Charta zur Gesundheitsförderung hat zum Thema „Befähigen und ermöglichen“ festgelegt: „Gesundheitsförderung ist auf Chancengleichheit auf dem Gebiet der Gesundheit gerichtet. Gesundheitsförderndes Handeln bemüht sich darum, bestehende soziale Unterschiede des Gesundheitszustandes zu verringern sowie gleiche Möglichkeiten und Voraussetzungen zu schaffen damit alle Menschen befähigt werden, ihr größtmögliches Gesundheitspotential zu verwirklichen. [...]" (WHO, Europa, 1986)

\section{Fragen und Methodik der Bachelorarbeit}

Folgende Fragen sollten mit meiner Bachelorarbeit beantwortet werden:

1. Welche Themen aus den Bereichen Gesundheitsprävention und häufige Erkrankungen sind in Leichter Sprache ausgearbeitet und stehen als Broschüre zur Verfügung? 
2. Erfüllen ausgewählte Broschüren die Kriterien von Leichter Sprache und genügen sie den allgemeinen Anforderungen der,Wittener Liste'?

3. Weisen ausgewählte Broschüren in Leichter Sprache und Standard-Sprache Unterschiede auf?

Die Methodik der Bachelorarbeit gliederte sich in vier Teilbereiche:

\section{Systematische Literaturrecherche}

Die systematische Literaturrecherche wurde in den wissenschaftlichen Datenbanken Livivio, Cinahl, psychiatry online und Pubmed durchgeführt. Es wurden Studien im Umfeld von Leichter Sprache gesucht. Der Fokus lag dabei auf Studien zu geistig behinderten Menschen im Hinblick auf Gesundheitsprävention und chronischen Erkrankungen, wie sie auch bei der allgemeinen Bevölkerung vorkommen. Eingeschlossen wurden Studien, die sich mit schriftlichem Informationsmaterial für Erwachsene zu den Themen Gesundheitsprävention und Behandlung von häufigen Erkrankungen beschäftigt haben. Ausgeschlossen wurden mündliche Beratungen/Schulungen, Informationen durch Filme, Kinder als Zielgruppe, Alphabetisierung von Risikogruppen und Studien, die älter als 10 Jahre waren.

\section{Broschürensuche}

Die Broschürensuche wurde auf deutschsprachigen Internetseiten durchgeführt, auf denen Broschüren bestellt werden konnten, die in Leichter Sprache verfasst sind. Es sollte ein realistisches Bild für die Zugänglichkeit von Bro-

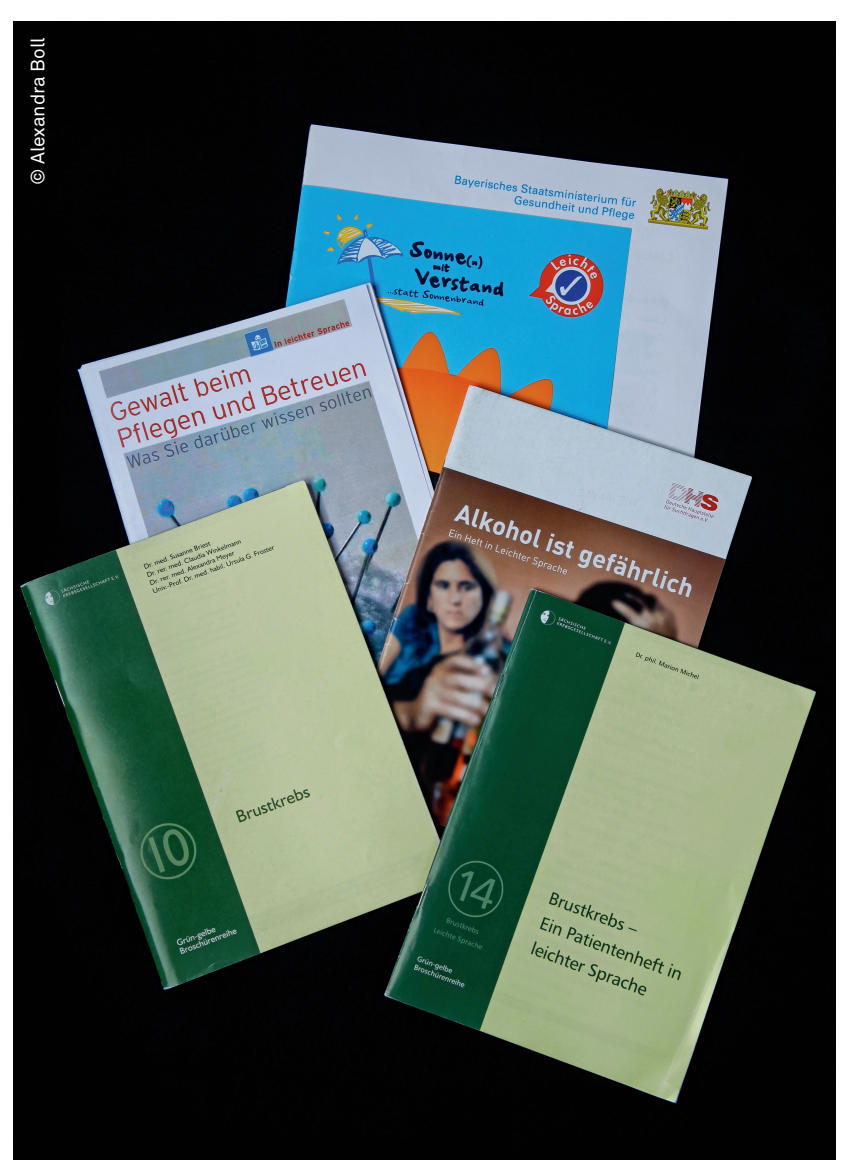

schüren in Deutschland erarbeitet werden. Die Suche nach Broschüren als Print-Medium war Ziel der Arbeit, da diese mehrere Vorteile bieten:

- Betroffene können die Broschüre selbst in die Hand nehmen und sie zwischendurch zur Seite legen ohne erneut den Computer einschalten und die entsprechende Internetseite suchen zu müssen.

- Betreuende, Pflegende, Ärzte, Therapeuten, gesetzliche Vertreter und Angehörige können mit den Betroffenen zusammen das Informationsmaterial besprechen.

- Zu einem späteren Zeitpunkt können die Informationsmaterialien erneut in die Hand genommen und Inhalte wiederholt gelesen oder besprochen werden.

\section{Recherche und Auswahl eines Konzeptes zur Beurteilung der Broschüren}

Für die Leichte Sprache wurden die verfügbaren Regelwerke miteinander verglichen und auf Gemeinsamkeiten und Unterschiedlichkeiten hin geprüft. Auch die Entstehungsgeschichte der Konzepte wurde analysiert, insbesondere ob die Regelwerke mit den betroffenen Menschen im Sinne der Partizipation entwickelt und konzipiert wurden. Desweiteren sollte herausgefunden werden, ob die Konzepte wissenschaftlich evaluiert wurden. Die Recherche wurde im Internet und per Freihandsuche durchgeführt und abschließend ein Konzept zur Bewertung der Broschüren ausgewählt. Dies wurde anhand der vorgenannten Kritieren vorgenommen, sowie hinsichtlich der Praktikabilität bei der Er- und Bearbeitung von Texten.

\section{Beurteilung exemplarischer Broschüren}

Die vorliegenden Broschüren wurden exemplarisch anhand der ,Wittener Liste' beurteilt. Für die Frage nach der Verständlichkeit wurde das Hamburger Verständlichkeitskonzept (Langer et al., 2015) hinzugezogen. Es gilt umfassender als Lesbarkeitsformeln und schätzt folgende Kriterien ein: Einfachheit, Gliederung/Ordnung, Kürze / Prägnanz und Anregende Zusätze.

Die ,Wittener Liste' ist zur Beurteilung von Broschüren durch Pflegende im Umfeld der Aktivitäten des „Netzwerk Patienten- und Familienedukation in der Pflege e.V.“ entstanden. Sie stützt sich auf Fachliteratur und gängige Verfahren zur Qualitätssicherung medizinischer Patienteninformationen. Sie enthält folgende Kriterien:Zielgruppe / Ziel vorhanden, Pflege-und Alltagsbezug (Relevanz), Ermutigung/persönliche Ansprache, Umfang und Schrittgröße, Verständlichkeit, Layout, Wissensstützung/Quellenangabe, Autorenhinweise / Finanzierung, weiterführende Adressen, Vollständigkeit.

Für die Broschüren in Leichter Sprache wurde das Regelwerk des ,Netzwerk Leichte Sprache‘ zugrundegelegt, hier müssen alle Regeln vollständig eingehalten werden.

\section{Theoretischer Hintergrund}

Als Patientenedukation wird die Schulung, Information und Beratung von Patienten (und Angehörigen) bezeich- 


\section{Regelwerk des ,Netzwerk Leichte Sprache“}

1. Thema genau erklären, ebenso Fremdwörter und Fachbegriffe.

- Menschen mit Lernschwierigkeiten beim Schreiben von Texten einbeziehen.

- Leicht verständliche, allgemein bekannte Wörter verwenden.

- Im ganzen Text dasselbe Wort für dieselbe Sache verwenden.

- Keine Metaphern verwenden.

- Keine Abkürzungen verwenden oder aber erklären.

- Möglichst keine Prozent-Zahlen oder hohe Zahlen verwenden.

- Immer kurze Sätze schreiben.

- Direkte Ansprache und Ansprache mit „Sie“.

- Verneinungen vermeiden.

- Nach Möglichkeit aktive Formen verwenden und passive Formen vermeiden. (Bsp.: „Peter hat die Besprechung abgesagt.“ Anstelle von „Die Besprechung wurde abgesagt.“)

- Dokumente so gestalten, dass es leicht zu lesen und zu verstehen ist. Zeilen-Abstand 1,5.

- Keinen Hintergrund verwenden, der es schwer macht, den Text zu lesen.

- Klare und leicht lesbare Schrift-Art verwenden (keine Serifen, eng zusammen stehende Buchstaben, zu dünn, kursive, spezielle oder bunte Schriften, keine Unterstreichungen)

- Wenn möglich, keine Sonder-Zeichen verwenden.

- Mit einem neuen Satz in einer neuen Zeile beginnen.

- Keine Wort-Trennungen am Zeilen-Ende.

- Leicht verständliche Überschriften, die klar machen, worum es im Text geht.

- Die wichtigste Information sollte leicht zu finden sein: am Anfang des Textes, fett drucken oder ein eingerahmtes Feld nutzen.

- Linksbündig schreiben. Keinen Blocksatz verwenden.

- Bilder verwenden, um zu erklären, um was es im Text geht.

- Bilder verwenden, die klar und leicht verständlich sind, bzw. eindeutig zum Text passen.

- Keine zu langen Wörter verwenden oder mit Binde-Strichen trennen.

- Verben verwenden.

- Kein Genitiv.

- Nur eine Information pro Satz.

- Kein Konjunktiv.

- Wörter benutzen, die etwas genau beschreiben.

- Arabische Zahlen verwenden. Keine römischen.

- Alte Jahreszahlen vermeiden.

- Zahlen als Ziffern schreiben.

- Datum: 3. März 2016 oder 3.3.2016

- Telefon-Nummern mit Leer-Zeichen schreiben.

- Einfachen Satzbau verwenden.

- Fragen im Text vermeiden.

- Quer-Verweise vermeiden. Alles zusammen schreiben, was zusammen gehört.

- Adressen wie auf einem Brief schreiben.

- Dunkle Schrift und helles Papier bevorzugen.

- Dickes und mattes Papier verwenden. Mind. 80 Gramm.

net. Sie dient der Gesundheitsförderung und soll bei der Bewältigung des Alltags mit einer chronischen Erkrankung helfen. Sie kann mit Hilfe von Filmen, Büchern, Gesprächen und Broschüren durchgeführt werden. Informationsbroschüren haben in der Patientenedukation schon lange Tradition. Die Broschüre kann als Leitfaden für ein strukturiertes Beratungsgespräch genutzt werden, damit nichts Wichtiges vergessen wird. Am Ende des Gespräches kann die Broschüre als Informationsmaterial in den Hän- den des Betroffenen bleiben und noch einmal nachgelesen und erinnert werden. Außerdem kann diese an Angehörige des Betroffenen weiter gereicht werden, damit sie ebenfalls informiert sind und den Betroffenen unterstützen können. Nach längerer Zeit dient sie als Erinnerungsbrücke, z.B. wenn eine Neuorientierung durchgeführt und aufrecht erhalten werden sollte. Die Broschüren sind so zu gestalten, dass sie für die Adressaten leicht lesbar, verstehbar und anschaulich dargestellt sind. 


\section{Menschen mit geistiger Behinderung}

Die Weltgesundheitsorganisation definiert den Begriff „geistige Behinderung“ folgendermaßen: „Geistige Behinderung bedeutet eine signifikant verringerte Fähigkeit, neue oder komplexe Informationen zu verstehen und neue Fähigkeiten zu erlernen und anzuwenden (beeinträchtigte Intelligenz). Dadurch verringert sich die Fähigkeit, ein unabhängiges Leben zu führen (beeinträchtigte soziale Kompetenz). Dieser Prozess beginnt vor dem Erwachsenenalter und hat dauerhafte Auswirkungen auf die Entwicklung." (WHO, Europa, 2016) Die Lernbehinderungen äußern sich häufig durch Schwierigkeiten beim Lesen, Verstehen und Kommunizieren.

Geistig behinderte Menschen erreichen immer häufiger auch ein hohes Lebensalter, ähnlich wie Menschen ohne Behinderung. Dadurch sind sie ebenfalls durch chronische Erkrankungen und Multimorbidität bedroht. Gesundheitspräventive Maßnahmen und eine frühzeitige Behandlung von chronischen Erkrankungen sind deshalb besonders wichtig.

\section{Gesundheitsprävention und häufige Erkrankungen}

Gesundheitspräventive Maßnahmen dienen zur Erhaltung der Gesundheit durch Ausschaltung von gesundheitsschädigenden Faktoren. Sekundäre Prävention wird durch Vorsorgeuntersuchungen zur Sicherstellung frühestmöglicher Diagnose und Therapie ausgeübt. (Pschyrembel, 1994) Angebote zu den Themen Ernährung, Bewegung, Stress und Sucht werden gesundheitspolitisch den präventiven Maßnahmen zugeordnet und gefördert.

Da Behinderung nicht mit Krankheit gleichzusetzen ist - ein behinderter Mensch kann trotz Behinderung gesund sein - gilt es, die Gesundheit so lange wie möglich durch gesundheitspräventive Maßnahmen zu erhalten.

Eine akute oder chronische Erkrankung kann in jedem Lebensalter auftreten. Durch das Erreichen höherer Lebensalter, steigt die Gefahr chronisch an einer koronaren Herzkrankheit, Schlaganfall, Diabetes, Krebs oder Atemwegs-Erkrankungen zu erkranken. (RKI 2014)

Diese Krankheiten sollten im Sinne der sekundären Prävention früh erkannt und behandelt werden, um einer Multimorbidität vorzubeugen. Ist eine Erkrankung bereits eingetreten, so sollte es dem (geistig) behinderten Menschen ermöglicht werden, aktiv dazu beitragen zu können, die Gesundheit vollständig oder teilweise wieder zu erlangen.

\section{Definitionen, Ursprung und Regeln zur Leichten Sprache}

Die meisten Definitionen beziehen sich auf das Lesen und Schreiben, sowie das Verstehen von Texten. Beispiel der Inclusion Europe: „These standards are to help people make their information easy to read and understand." Lediglich zwei thematisieren auch das Sprechen in Leichter Sprache: die Definition des ,Netzwerkes Leichte Sprache und des Hurraki-Wörterbuches.

Bei meiner Recherche konnte ich insgesamt acht Konzepte für Leichte Sprache finden. Ein internationales von der „International Federationof Library AssociationandInstitutions“ (IFLA) und zwei europäische von „Inclusion Europe“, mit 9 Partner-Ländern, sowie vom ,Netzwerk Leichte Sprache' mit 5 Mitgliedsländern. Auf nationaler Ebene konnten die Regelwerke der Lebenshilfe Bremen, des Bundesministerium für Arbeit und Soziales, des Franchise-Unternehmen Capito, der Forschungsstelle Leichte Sprache an der Universität Hildesheim sowie die Barriere freie-Informationstechnik-Verordnung (BITV 2.0) identifiziert werden.

Für die Broschüren-Beurteilung in der Bachelorarbeit wurde das gemeinsame Regelwerk des, Netzwerk Leichte Sprache', Lebenshilfe Bremen und des Bundesministeriums für Arbeit und Soziales zugrunde gelegt.

Begründung:

1. Das Regelwerk beinhaltet 39 Fragen, die weder zu gering, noch zu viel erscheinen. Die Praktikabilität bei der

Erstellung von Texten ist dadurch gegeben.

2. Das Regelwerk ist in Deutschland das Gebräuchlichste.

3. Das Regelwerk wurde aus der Praxis heraus mit der Zielgruppe zusammen entwickelt.

4. Die Regeln sehen vor, dass die Zielgruppe eine abschließende Prüfung des Textes vornimmt.

\section{Systematische Literaturrecherche}

An einer Studie von Dowse et al. (2014) nahmen in Südafrika 116 HIV-Patienten teil. 58 HIV-Patienten erhielten eine Patienteninformation, die einfach formuliert und mit Bildern illustriert war. Die randomisiert kontrollierte Studie führte zu Beginn und nach 1, 3 und 6 Monaten einen Test mit 22 Fragen durch. Hierzu wurden mit allen Patienten Interviews geführt und Fragen zu den Kenntnissen über die antivirale Therapie mit Wirkweise, Nebenwirkungen und Zusammenhänge zwischen HIV und Aids gestellt. Zusätzlich sollten die Patienten anhand einer Selbstwirksamkeitsskala die Einhaltung ihrer Behandlung einschätzen. Es konnten signifikante Unterschiede in der Interventionsgruppe festgestellt werden. Sie hatten nach 6 Monaten deutlich mehr Kenntnisse zu:

- Medikamenten-Namen und Dosierung,

- den Nebenwirkungen,

- den Lagerungsbedingungen,

- der lebenslang notwendigen Tabletten-Einnahme,

- sowie Kenntnisse über die Ausbreitung von HIV und

- den Ausbruch der Erkrankung Aids.

Ryan et al. (2014) haben 97 Patienteninformationen analysiert. Die Studie wurde in den USA an einem Klinikzentrum in drei unterschiedlichen Abteilungen durchgeführt: Krebszentrum, Abteilung für Schlaganfall-Patienten sowie in einer Mutter-Kind-Station. Die Lesbarkeit der schriftlichen Informationsmaterialien wurde anhand von sieben Lesegraden beurteilt. Hierzu wurde eine Software (readabilityconcensuscalculator) genutzt, die die Anzahl der Sätze, Wörter, Silben und Zeichen auswertet. Der Lesegrad 5 bedeutet eine hervorragende Lesbarkeit, die Grade $6-8$ bedeuten eine adäquate Lesbarkeit und ab Grad 9 ist der Text schwierig zu lesen. 
Tabelle 1. Ergebnis der Kriterien der Wittener Liste und den Regeln der Leichten Sprache bei drei Broschüren. (eigene Darstellung)

\begin{tabular}{lcl}
\hline Broschüre & Kriterien der ,Wittener Liste & Regeln der Leichten Sprache \\
\hline Gewalt beim Pflegen und Betreuen & 8 von 13 & 34 von 37 \\
Alkohol ist gefährlich & 10 von 13 & 29 von 37 \\
Sonne(n) mit Verstand ... statt Sonnenbrand & 5 von 13 & 27 von 37 \\
\hline
\end{tabular}

Die Informationsmaterialien wurden mit der SAM-Skala (Suitability Assessment of Materials tool) auch auf die Verständlichkeit sowie Lesbarkeit hin überprüft.

Im Ergebnis wurden beide Eigenschaften bewertet und zusammengefasst. Insgesamt wurden nur 7 von 97 Broschüren (ca. 7\%) für Patienten mit Leseschwierigkeiten als geeignet bewertet. Die Autoren ziehen das Fazit, dass es zur Verbesserung von Behandlungsergebnissen sowie zum Ausgleich von gesundheitlichen Unterschieden zwischen den Bevölkerungsschichten wichtig ist, dass die Patienten kohärent mit ihrer Erkrankung umgehen können. Leicht verständliches Informationsmaterial kann einen Teil dazu beitragen, besonders für Patienten und deren Familien, die Leseschwierigkeiten haben.

\section{Ergebnisse der Broschüren-Suche}

Insgesamt konnten elf Broschüren in Leichter Sprache gefunden und kostenlos bestellt oder herunter geladen werden.

Es wurden drei Broschüren ausgewählt, die mit einem Prüfsiegel eines Leichte Sprache-Konzeptes versehen bzw. von einem Büro für Leichte Sprache übersetzt wurden. Alle wurden anhand der ,Wittener Liste' sowie dem ausgewählten Regelwerk für Leichte Sprache bewertet:

- Die Themen der drei Broschüren sind für den Bereich der Gesundheitsprävention Alltags-relevant.

- Die äußere Ansicht ist bei allen Broschüren ansprechend und farbig gestaltet. Alle sind durch einen einfachen Satzbau überwiegend leicht zu lesen.

- Die Texte werden jeweils mit Bildern ergänzt und sind nicht zu umfangreich. Sie sind jeweils durch Prüfer für Leichte Sprache überprüft worden.

- Nur eine Broschüre verfügt über ein Inhaltsverzeichnis.

- Zwei kann man durch ein Logo der Leichten Sprache sofort als Broschüren in Leichter Sprache erkennen. Es wurde einmal das Logo der „Inclusioneurope“ und einmal des „Netzwerkes Leichte Sprache“ verwendet. Die dritte Broschüre verfügt über kein Logo, obwohl die Ersteller Mitglied im ,Netzwerk Leichte Sprache sind.

- Alle drei Broschüren haben keine Literaturangaben gemacht und in zwei Broschüren fehlen zur Vollständigkeit relevante Inhalte.

- Der Leser wird überwiegend persönlich angesprochen und alle verfügen über mindestens eine weiterführende Adresse.

Für die Darstellung in Tabelle 1 wurden die 10 Kriterien der ,Wittener Liste، incl. des Hamburger Verständlichkeitskonzeptes mit 4 Kriterien zugrunde gelegt. Der 5. Punkt der ,Wittener Liste' (Verständlichkeit) wurde also durch 4 Kriterien ersetzt, sodass sich 13 Bewertungskriterien ergeben. Bei den Regeln der Leichten Sprache wurden 2 Regeln nicht eingeschlossen, da sie in allen Broschüren nicht vorgekommen sind (Alte Jahreszahlen vermeiden / Datum).

Zwei weitere Broschüren, eine in Leichter und eine in Standard-Sprache zum gleichen Thema, wurden anhand der,Wittener Liste' bewertet und miteinander verglichen. Punkt 5. der ,Wittener Liste' wurde bei der Broschüre in Leichter Sprache durch die Regeln der Leichten Sprache und bei der Broschüre in Standard-Sprache anhand des Hamburger Verständlichkeitskonzeptes geprüft.

Die äußeren Ansichten der beiden Exemplare unterscheiden sich kaum.

Die Autorenhinweise auf der 1. Seite der Broschüre in Leichter Sprache sind um die Angaben der Übersetzung ergänzt.

Die beiden Inhaltsverzeichnisse unterscheiden sich deutlich: die Broschüre in Leichter Sprache hat knapp doppelt so viele Kapitel, ist also differenzierter aufgeteilt.

Am Ende der Broschüre in Leichter Sprache sind alle wichtigen Worte kurz erklärt. Die andere hat keinen Glossar, dafür aber einen Anhang mit weiterführenden Kontaktadressen.

Beide haben in der Mitte einen doppelseitigen Spendenaufruf und einen Mitgliedsantrag für den Beitritt zur Sächsischen Krebsgesellschaft e.V.

Beiden Exemplaren gemein ist, dass sie einen viel $\mathrm{zu}$ großen Umfang haben und somit eigentlich keine Broschüren mehr sind, sondern Hefte.

Außerdem werden in beiden Broschüren keine Literaturhinweise gemacht und die verwendeten Bilder sind entweder in schlechter Qualität und Farbdarstellung und/oder teilweise zu klein.

In der Broschüre Leichte Sprache sind immer wieder farbige Kästchen eingefügt. Dort kann der Leser Fragen eintragen, welche er beim nächsten Arztbesuch noch stellen möchte. Diese Notizenkästchen sind im anderen Heft nicht zu finden.

Es gibt sehr viele verschachtelte und lange Sätze in der Broschüre in Standard-Sprache, die für einen Laien schwer verständlich sind. Dagegen lassen sich die Texte in Leichter Sprache einfach lesen und verstehen.

Inhaltlich unterscheiden sich die beiden Broschüren sehr deutlich. Zum einen werden einige Themen in Leichter Sprache gar nicht angesprochen, zum anderen wird Vieles nur kurz und knapp erläutert. Weiter führende Informationen soll sich der Leser dann beim behandelnden Arzt einholen. 
Tabelle 2. Vergleich einer Broschüre in Leichter Sprache mit einer in Standard Sprache

\begin{tabular}{lll}
\hline Broschüre & Kriterien der, Wittener Liste & Regeln der Leichten Sprache \\
\hline $\begin{array}{l}\text { Brustkrebs - Ein Patientenheft in leichter } \\
\text { Sprache }\end{array}$ & 3 von 13 & 29 von 37 \\
Brustkrebs & 3 von 13 &. \\
\hline
\end{tabular}

Viele Themen, die in Standard-Sprache ausführlich beschrieben wurden, sind in der Broschüre in Leichter Sprache nicht zu finden.

Themen die in der Broschüre in Leichter Sprache benannt sind, aber in Standard-Sprache nicht, sind folgende:

- „Z.B. können Sie eine Kur bekommen.

- Oder einen Schwer-Behinderten-Ausweis.

- Wenn Sie nicht mehr arbeiten können, stellen Sie einen Antrag damit Sie Rente bekommen."

Folgende Kapitel sind in der Broschüre in StandardSprache ausführlich beschrieben, in der in Leichter Sprache aber nur mit wenigen Sätzen / Worten erläutert. Nachfolgend drei Beispiele:

- „Warum gerade Ich? Risikofaktoren“: In Standard-Sprache ist dies mit 419 Wörtern ausgeführt, im Vergleich zu „Sind Sie selbst schuld, wenn sie Brust-Krebs bekommen?" in Leichter Sprache mit 136 Wörtern.

- Dem Thema „Computertomografie (CT)“ ist ein eigenes Kapitel in Standard-Sprache mit 86 Wörtern gewidmet, in Leichter Sprache sind es 5 Sätze mit 35 Wörtern.

- „Ernährung“ hat in Standard-Sprache 245 Wörter und in Leichter Sprache 29 Wörter.

Die Nachsorge-Dauer nach der Brustkrebs-Operation wird in der Broschüre in Leichter Sprache mit 5 Jahren angegeben, in der anderen mit 10 Jahren!

Nebenwirkungen der unterschiedlichen Behandlungsarten (Chemotherapie, Bestrahlung, etc.) werden in Leichter Sprache deutlich weniger benannt, in Standard-Sprache sehr ausführlich beschrieben.

In der Broschüre in Standard-Sprache werden sehr viele Fachbegriffe verwendet. Diese werden größtenteils kurz erklärt. In der anderen Broschüre werden wenige Fachbegriffe verwendet und meist ausführlich erklärt.

Bilder, Grafiken, Zeichnungen und Tabellen sind in beiden Broschüren meistens sehr klein dargestellt.

Für die Darstellung in Tabelle 2 wurden die 10 Kriterien der ,Wittener Liste' incl. des Hamburger Verständlichkeitskonzeptes mit 4 Kriterien zugrunde gelegt. Der 5. Punkt der ,Wittener Liste' (Verständlichkeit) wurde also durch 4 Kriterien ersetzt, sodass sich 13 Bewertungskriterien ergeben. Bei den Regeln der Leichten Sprache wurden 2 Regeln nicht eingeschlossen, da sie in allen Broschüren nicht vorgekommen sind (Alte Jahreszahlen vermeiden/Datum).

\section{Zusammenfassung der Ergebnisse}

Bezogen auf die Ausgangsfragen kann festgestellt werden, dass nur in einem geringen Umfang Broschüren in Leich- ter Sprache zur Gesundheitsprävention bzw. zu häufigen Erkrankungen zur Verfügung stehen.

Insgesamt konnten 11 Publikationen bestellt werden, wovon 5 das Format eines Flyers und 2 den Umfang eines Heftes haben.

Nur zwei Broschüren waren mit einem Logo für Leichte Sprache versehen.

Zusammengefasst kann die zweite Frage der Bachelorarbeit wie folgt beantwortet werden: die Broschüren erfüllen in einer relativ hohen Ausprägung zwar die Kriterien von Leichter Sprache, genügen aber weniger den Anforderungen der ,Wittener Liste.

Die dritte Frage dieser Arbeit kann nur limitiert anhand von zwei Broschüren beantwortet werden: Es gab große inhaltliche Unterschiede.

\section{Diskussion}

Insgesamt verdeutlicht diese Arbeit, dass es nur sehr wenige Informationsmaterialien in Leichter Sprache für die beiden Themenbereiche gibt. Dies wirft die Frage auf, ob Menschen mit einer geistigen Behinderung ihre Selbstbestimmungsrechte und Teilhabe ausreichend in Anspruch nehmen können.

Die Kriterien der ,Wittener Liste', die als Maßstab für die inhaltliche Qualität der Patienteninformationen angesehen werden können, wurden bei allen 5 Broschüren nicht vollständig erfüllt. Das Minimum lag bei 3 und das Maximum bei 10 vollständig eingehaltenen Kriterien. Für den Umgang mit der eigenen Gesundheit oder Erkrankung ist es wichtig, vollständiges, alltagsbezogenes, verständliches, neuzeitliches, unabhängiges und positiv formuliertes Wissen zu erlangen.

Die Regeln der Leichten Sprache wurden umfangreicher als die Kriterien der ,Wittener Liste' eingehalten. Der geringste Wert lag bei 27, der höchste bei 34 der vollständig eingehaltenen Regeln. Dass diese nicht $100 \%$ ig eingehalten wurden, erscheint zumindest bei zwei Broschüren bizarr: die Broschüren „Gewalt beim Pflegen und Betreuen“ sowie „Sonne(n) mit Verstand ... statt Sonnenbrand“ sind jeweils mit einem Prüfsiegel versehen. Diese dürfen nur verwendet werden, wenn sie von ausgebildeten Fachleuten anhand der Regelwerke erstellt und von der Zielgruppe überprüft worden sind. Das hier zur Überprüfung verwendete Regelwerk ist dasselbe, wie das bei der Broschüren-Erstellung. Ob die Kenntlichmachung mit einem Prüfsiegel / Logo für eine hohe Qualität der Leichten Sprache garantiert, wird hinterfragt.

Eine Patienteninformation in Leichter Sprache sollte Beides beinhalten:

- fachliche Korrektheit und

- hohe Qualität der Leichten Sprache 
damit sie gezielt und gut informieren kann. Dies kann durch den Einsatz von den beiden Überprüfungsinstrumenten, der ,Wittener Liste' und einem Regelwerk für Leichte Sprache erreicht werden. Ähnliche Hinweise liefert auch die Studie von Ryan et al. (2014). Hier wurden ebenfalls zwei Instrumente genutzt, das ,Suitability Assessment of Materials tool' und der ,Text Readability Consensus Calculator'. Ideal wäre die Entwicklung eines Instrumentes, mit welchem Patienteninformation in Leichter Sprache erstellt bzw. überprüft werden könnten.

Generell bleibt die Frage offen, wer eine Broschüre erstellen soll. Fachexperten, wie Mediziner, Wissenschaftler oder Juristen, beherrschen in der Regel die Leichte Sprache nicht. Prüfer der Zielgruppe und Übersetzer für Leichte Sprache verfügen nicht über notwendige Fachkompetenzen. Diesem Dilemma kann vermutlich nur mit intensiver Zusammenarbeit und mehrmaligen Prüfvorgängen begegnet werden. Dass dies erfolgreich sein kann, zeigt die Studie von Dowse et al. (2014).

Ob eine Überprüfung durch eine Fachsoftware, wie es die Universität Hildesheim praktiziert, zu korrekteren Ergebnissen führt, müsste Gegenstand weiterer Untersuchungen werden.

Möglicherweise reicht es auch aus, für Patienten-Informationsbroschüren die ,Wittener Liste ' und ein Regelwerk für Leichte Sprache miteinander zu verknüpfen. Für andere Informationsbroschüren müssten die entsprechenden Fachexperten eigene Kriterien konzipieren und sie mit Leichte Sprache-Regeln ergänzen.

$\mathrm{Ob}$ es Antworten auf diese Fragen aus dem europäischen oder internationalen Raum gibt, könnte durch eine weitere Recherche eruiert werden.

Die inhaltlichen Unterschiede beim Vergleich der zwei Broschüren zum Thema Brustkrebs werfen weitere Fragen auf. Hätten die Angaben in der Broschüre in Leichter Sprache auch für die in Standard-Sprache ausgereicht? Möglicherweise wäre es hilfreicher gewesen, mehrere Themenblöcke in separaten Broschüren zu behandeln. Nützen Hinweise, beim nächsten Besuch dem behandelnden Arzt Fragen zu stellen, wenn dieser die Leichte Sprache nicht beherrscht? Zur Beantwortung müssten weitere Broschüren miteinander verglichen und mit der Zielgruppe besprochen und praktisch getestet werden.

Zum methodischen Vorgehen: Möglicherweise wurden bei der Recherche noch weitere Internetseiten übersehen, auf denen ebenfalls Broschüren hätten bestellt werden können.

Unter Umständen stehen weitere Broschüren in Leichter Sprache zur Verfügung, die aber nicht über das Internet bestellt werden konnten. Eine Suche bzw. Anfrage in Arztpraxen, Krankenhäusern oder Beratungsstellen wurde nicht durchgeführt.

Eine zusätzliche Recherche in den Ländern, die an der Erstellung der einbezogenen Regelwerke beteiligt waren, hätte unter Umständen ebenfalls eine größere Anzahl an Broschüren hervorgebracht. Ob die Broschüren auf die Verhältnisse in Deutschland passen würden, ist fragwür- dig. Auf andere Länder können die Ergebnisse dieser Arbeit nicht übertragen werden.

\section{Fazit}

Broschüren zur Gesundheitsprävention und zu häufigen Erkrankungen sollten flächendeckend auch für Menschen mit Lernschwierigkeiten zur Verfügung stehen. Bisher ist dies nicht der Fall. Es könnte Menschen mit geistiger Behinderung helfen, sich besser über gesundheitspräventive Maßnahmen oder der Behandlung ihrer Erkrankung zu informieren. Ebenso könnte es ihren Angehörigen, pflegerischen oder pädagogischen Betreuern, Therapeuten und Ärzten helfen, den Betroffenen leicht verständlich zu informieren und zu beraten.

Gut wäre die Entwicklung einer Einschätzungshilfe, welche bei der Erstellung und/oder Überprüfung von kontextspezifischen Broschüren in Leichter Sprache Hilfe bietet - gut wäre auch die Zusammenarbeit zwischen Experten/Innen für Leichte Sprache und Experten/Innen für Broschüren in der Patientenedukation. Für Pflegelehrer/Innen aller Bildungsstufen gilt die Anregung, mit Studierenden/Auszubildenden eine Patientenbroschüre in Leichte Sprache umzuwandeln.

\section{Literatur}

Bundesministerium für Arbeit und Soziales: Begründung zur Barrierefreien-Informationstechnik-Verordnung, BITV 2.0, 2011. http://www.einfach-teilhaben.de/SharedDocs/Downloads/DE/ StdS/Aktuelles/bitv_begruendung. pdf?__blob=publicationFile [Zugriff: 02.12.16].

Bredel U., Maaß C. (2016) Leichte Sprache im Varietätengefüge des Deutschen. Leichte Sprache, Berlin: Duden.

Dowse R., Barford K., Browne S. H. (2014) Simple, illustrated medicines information improves ARV knowledge and patient selfefficacy in limited literacy South African HIV patients, AIDS Care, Vol. 26, 11: 1400-1406.

Inclusion Europe (2014) Information for all. http://easy-to-read. eu/wp-content/uploads/2014/12/EN_Information_for_all.pdf [Zugriff: 02.12.2016].

Langer I., Schulz von Thun F., Tausch R. (2015) Sich verständlich ausdrücken.10. Aufl. München: Ernst Reinhardt Verlag.

Maaß C. (2014) Forschungsstelle Leichte Sprache, Universität Hildesheim: Prüfsiegel Verständlichkeit,. https://www.uni-hildesheim.de/leichtesprache/pruefsiegel-siegel/pruefsiegel-verstaendlichkeit/ [Zugriff: 02.12.2016].

Netzwerk Leichte Sprache e.V. (2015) Das ist Leichte Sprache. http://www.leichtesprache.org/index.php/startseite/leichtesprache/das-ist-leichte-sprache [Zugriff: 02.12.2016].

Netzwerk Patienten- und Familienedukation in der Pflege e.V. (2014) Wittener Liste. http://patientenedukation.de/materialien/broschuerenbewertung [Zugriff: 02.12.2016].

Nomura M., Nielsen G. S., Tronbacke B. (2010) Guidelines for easyto-read materials / Revision on behalf of the IFLA/Library Services to People with Special Needs Section. The Hague, IFLA Headquarters. - 31 p. - 30 cm (IFLA Professional Reports; 120).

Pschyrembel (1994). Klinisches Wörterbuch. Hamburg: Nikol Verlagsgesellschaft $\mathrm{mbH}$.

Robert Koch Institut RKI (2014) Gesundheit in Deutschland aktuell 2012. http://www.rki.de/DE/Content/Gesundheitsmonitoring/ Gesundheitsberichterstattung/GBEDownloadsB/GEDA12. pdf?_blob=publicationFile [Zugriff: 02.12.2016]. 
Ryan L. et al. (2014). Evaluation of Printed Health Education Materials for Use by Low-Education Families. Journal of Nursing Scholarship, 46,4: 218-228.

WHO, Europa (2016) Definition des Begriffs „geistige Behinderung". http://www.euro.who.int/de/health-topics/noncommunicable-diseases/mental-health/news/news/2010/15/childrens-right-to-family-life/definition-intellectual-disability [Zugriff: 02.12.2016].

WHO, Europa (1986). Ottawa-Charta zur Gesundheitsförderung. http://www.euro.who.int/_data/assets/pdf_file/0006/129534/ Ottawa_Charter_G.pdf [Zugriff: 02.12.2016].

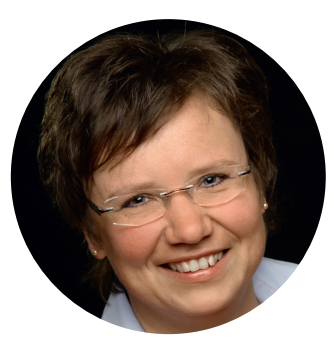

Alexandra Boll B.A.,

Altenpflegerin, Pflegedienstleitung, Fachwirtin in der Alten- und Krankenpflege (IHK) Qualitätsmanagement- und Hygiene-Beauftragte. Im Masterstudiengang „Versorgung von Menschen mit Demenz und chronischen Einschränkungen“

alexandra.boll@uni-wh.de 